\title{
Window of opportunity: A new insight into sequential bevacizumab and paclitaxel in two cases of metastatic triple-negative breast cancer
}

\author{
DAR-REN CHEN ${ }^{1,2}$, CHE LIN $^{1,3}$ and YU-FEN WANG ${ }^{2}$ \\ ${ }^{1}$ Comprehensive Breast Cancer Center; ${ }^{2}$ Cancer Research Center, Changhua Christian Hospital, Changhua 50006; \\ ${ }^{3}$ Department of Environmental Engineering, National Chung-Hsing University, Taichung 40227, Taiwan, R.O.C.
}

Received August 18, 2014; Accepted June 5, 2015

DOI: $10.3892 / \mathrm{etm} .2015 .2586$

\begin{abstract}
Bevacizumab, an antiangiogenic monoclonal antibody against vascular endothelial growth factor, was designed to normalize tumor vasculature and reduce intratumoral pressure. It can create a 'normalization window' during which the cancer can be attacked the most effectively, and the effects of chemotherapeutic drugs are enhanced. Representative trials (E2100, AVADO, RIBBON-1, RIBBON-2 and TURANDOT) have shown that the addition of bevacizumab to chemotherapy has significant benefits on progression-free survival for metastatic breast cancer, but not on overall survival. The present study describes two patients with metastatic triple-negative breast cancer who received 6 courses of bevacizumab-containing chemotherapy. Each course comprised $5-7.5 \mathrm{mg} / \mathrm{kg}$ bevacizumab administered on days 1 and 15 , and 20-24 h after bevacizumab delivery, $80 \mathrm{mg} / \mathrm{m}^{2}$ paclitaxel was administered for 3 weeks on days 2, 9 and 16, followed by 1 week of rest. Following sequential treatment with bevacizumab and paclitaxel, the results of computed tomography showed that the tumors were rapidly reduced in size. Based on the imaging findings from three-dimension power Doppler ultrasonography in one of the breast cancer patients who received neoadjuvant chemotherapy with bevacizumab, the possible timing of the normalization window was 20-24 h after the administration of bevacizumab. The normalization window may provide an opportunity to enhance the effect of chemotherapy with the aid of bevacizumab.
\end{abstract}

Correspondence to: Professor Dar-Ren Chen, Comprehensive Breast Cancer Center, Changhua Christian Hospital, 135 Nanhsiao Street, Changhua 50006, Taiwan, R.O.C

E-mail: darren_chen@cch.org.tw

Key words: bevacizumab, metastatic triple-negative breast cancer, paclitaxel, sequential treatment, vascular normalization

\section{Introduction}

In the E2100 trial, it was revealed that the initial treatment of metastatic breast cancer (MBC) with bevacizumab plus paclitaxel (BT regimen) greatly prolonged progression-free survival (PFS) from 5.9 to 11.8 months, but not overall survival (OS), as compared with paclitaxel alone (1). Other randomized controlled trials, AVADO (2) and RIBBON-1 (3), showed that the addition of bevacizumab to chemotherapy led to a statistically significant improvement in overall response and PFS in the first-line treatment of MBC. The subsequent trial TURANDOT (4) further demonstrated that the E2100 trial was not an outlier; it reproduced the considerable improvement of PFS by up to 11 months with the administration of weekly paclitaxel plus bevacizumab. All trials (E2100, AVADO, RIBBON-1, RIBBON-2 and TURANDOT) (1-5) showed significant PFS benefits for patients with MBC when bevacizumab was added to chemotherapy. However, PFS intervals were observed to differ among trials; furthermore adverse effects were reported including $1 \%$ mortality associated with bevacizumab (RIBBON-1 and AVADO).

Hypoxia is a key component in the regulation of angiogenesis (6). Vascular endothelial growth factor (VEGF) blockade can normalize tumor vasculature and reduce intratumoral pressure, thereby enhancing the effectiveness of chemotherapeutic drugs $(7,8)$. The changes in the normalization effects over time were further demonstrated by Vokoc et al, who observed that an anti-VEGF2 antibody can normalize tumor vasculature, which includes the pruning of immature vasculature, and the reduction of vessel permeability and intratumoral pressure (9). Since normalization is an important effect of anti-VEGF treatment, this raised the question of when the window of opportunity for concurrent chemotherapy is. According to the report by Winkler et al, this might be between 0 and 8 days in an animal model (10). Jain further characterized this period in mice with brain tumors, showing that the 'window open' state was achieved after 1 day of anti-VEGF treatment and that the 'window closed' at 5-6 days (11). We hypothesized that the infusion of bevacizumab 20-24 h prior to paclitaxel should provide the best window of opportunity and greatly enhance the effect of paclitaxel. Two cases were used to test this hypothesis. Written informed consent was obtained from 
the patients for publication of these case reports and accompanying images. The imaging findings of one of these cases, a breast cancer patient who received neoadjuvant chemotherapy, were used to show the possible timing of the window being open. To the best of our knowledge, this is the first report of the window of opportunity in human breast cancer.

\section{Case reports}

Case 1. An 85 year-old woman with metastatic triple negative breast cancer (TNBC) underwent left modified radical mastectomy. The pathology report revealed T3N3M0 and grade III TNBC. Seven months later, extensive chest wall recurrence with metastatic nodes in the right lower neck, left internal mammary chain, mediastinum and paratracheal region were found. Bevacizumab $(5-7.5 \mathrm{mg} / \mathrm{kg}$ ) was given on days 1 and 15 . Weekly paclitaxel was given $20-24 \mathrm{~h}$ after the delivery of bevacizumab at $80 \mathrm{mg} / \mathrm{m}^{2}$ (BT regimen); a marked effect on the resolution of the chest wall was observed with nearly half of the lesions disappearing within 7 days (Fig. 1A and B). One month later, the chest wall lesions were nearly cleared (Fig. 1C). The patient completed 6 courses of the BT regimen without adverse events such as hypertension and proteinuria, with the exception of 2 episodes of mild neutropenia.

Case 2. A 66-year-old woman was diagnosed with T2N1M0 TNBC following left modified radical mastectomy in 2004. Adjuvant chemotherapies were administered. The patient was diagnosed as having TNBC with minimal lung metastases in 2012. The patient received first line regimens of chemotherapy with vinorelbine. Two months later, the patient suffered from brain metastases with unstable gait and increased intracranial pressure syndrome. Following radiation therapy, the clinical symptoms were improved and size of the tumor in the brain decreased from $33.09 \mathrm{~mm}$ to $28.93 \mathrm{~mm}$. BT was delivered using the same protocol as described for Case 1. On days 1 and 15 , the patient was given $5-7.5 \mathrm{mg} / \mathrm{kg}$ bevacizumab; weekly paclitaxel $90 \mathrm{mg} / \mathrm{m}^{2}$ was given on days 2,9 and 16 . Prior to BT, the size of the tumor in the brain was $28.93 \mathrm{~mm}$, as detected by computed tomography (Fig. 2A). Following the delivery of the BT regimen, the tumor in the brain became smaller (Fig. 2B) and the patient underwent 6 courses of BT. Subsequently, metronomic therapy with 3-weekly sequential BT was administered. The patient was followed up 6 months later; the tumor in the brain remained a similar size (Fig. 2C). The patient tolerated this regimen for more than 1 year.

Under this regimen, both patients recovered within a short time with a satisfactory PFS. No grade 3-4 toxicities associated with bevacizumab were observed in the two patients. No cardiac toxicity was observed. Overall, the BT regimen was well tolerated, with manageable toxicities.

\section{Discussion}

In the era of chemotherapy and target therapy, it is important to identify responders and non-responders. There is no single regimen that is able to benefit all patients. Although high baseline plasma levels of VEGF-A and VEGFR-2 were found to be associated with a greater treatment effect in the AVEREL study (12) and proposed to be predictive markers
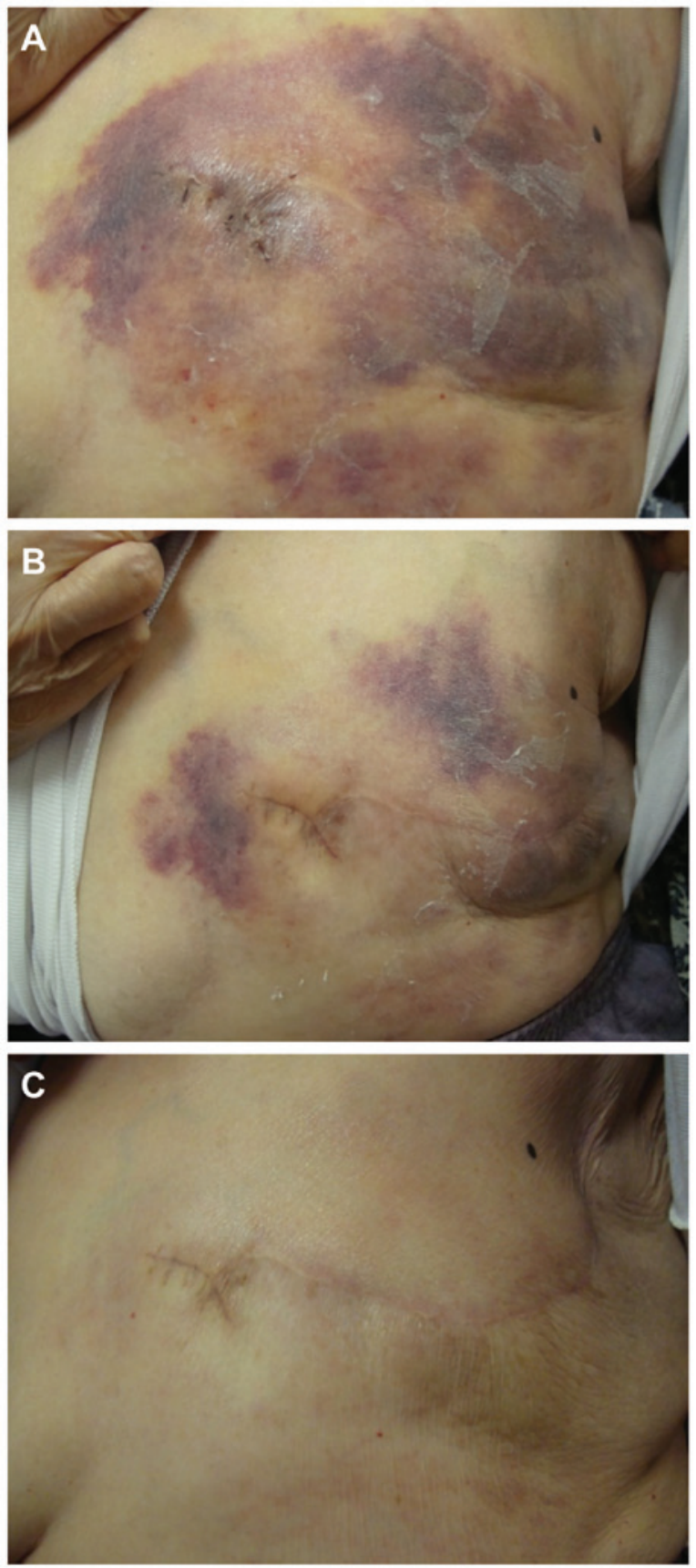

Figure 1. Case 1. Effects of sequential BT regimens on chest wall recurrence. (A) Extensive chest wall spreading of recurrent invasive ductal carcinoma (B) On day 7 after treatment, partial resolution was observed. (C) On day 28 , a nearly complete response was achieved. BT, bevacizumab and taxane (paclitaxel).

for bevacizumab efficacy in the retrospective analysis of the AVADO study (13), Hegde et al found that VEGF-A does not have predictive value for the benefits of bevacizumab-based treatment, at least for metastatic colorectal cancer, lung cancer and renal cell carcinoma (14). This controversial issue may be elucidated in the prospective MERiDiAN trial in the future.

The window of opportunity concept was introduced by Jain (11) on the basis of animal studies and relates to the taming of vessels to treat cancer. Studies of murine and human tumors have identified that the onset of normalization is typically 1-2 days after the commencement of therapy, followed 

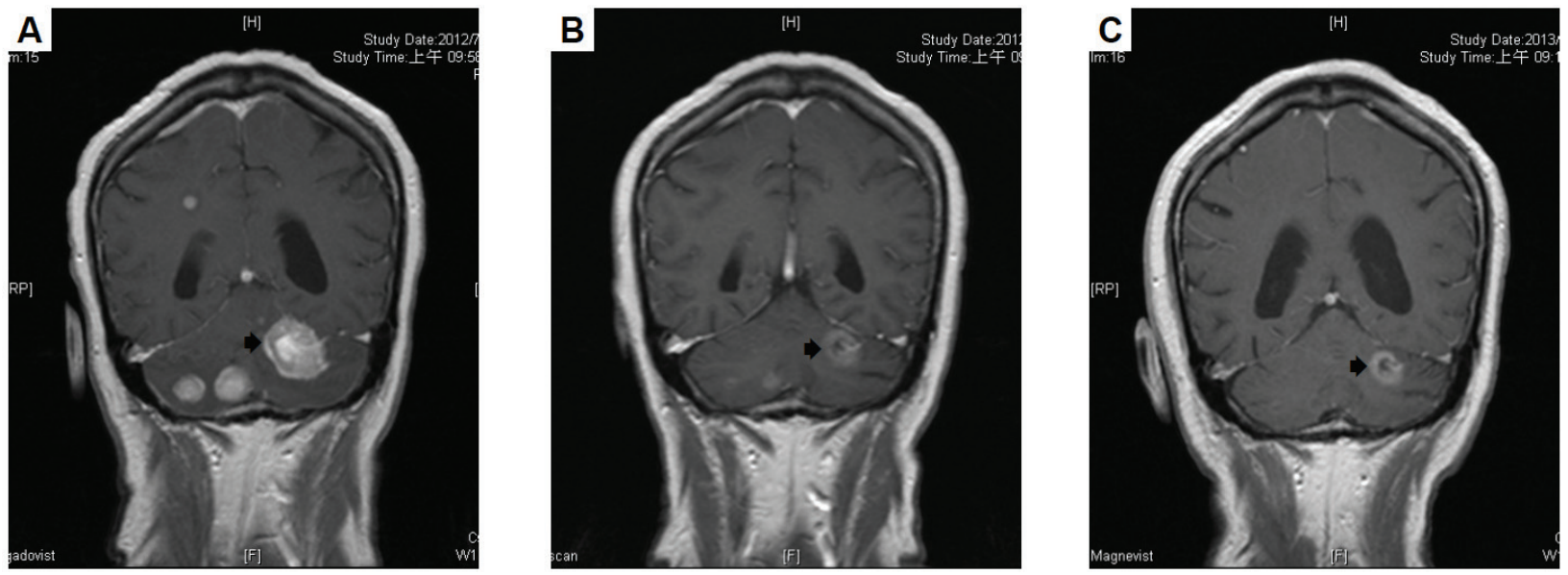

Figure 2. Tumor size change was detected using computed tomography prior to and following a sequential bevacizumab plus paclitaxel (BT) regimen. (A) Prior to treatment, the tumor size in brain was $28.93 \mathrm{~mm}$. (B) After 11 weeks of treatment, the size of the tumor in brain decreased. (C) At 6 months after completing 6 courses of BT regimen, the size of the tumor was further reduced.
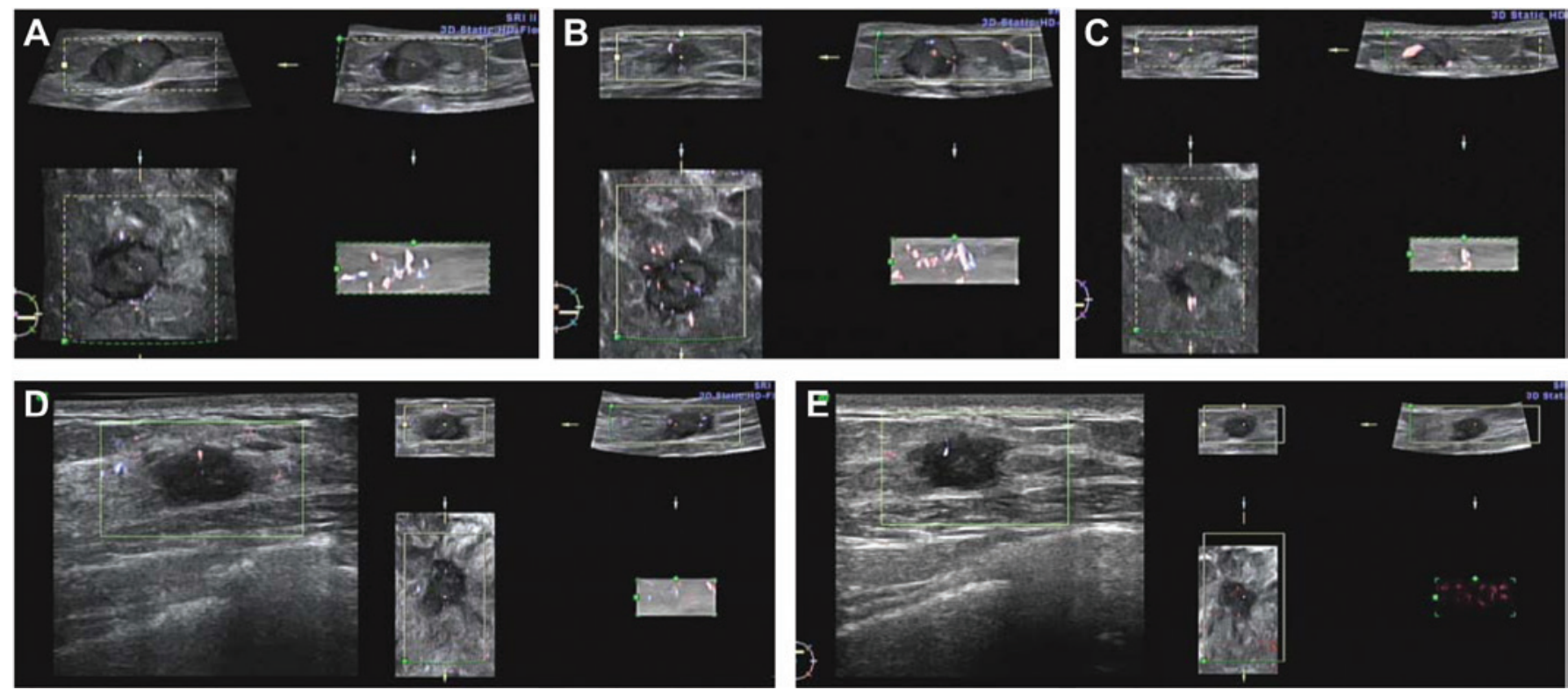

Figure 3. Normalization of tumor vasculature prior to and following neoadjuvant chemotherapy with bevacizumab. Three-dimension power Doppler ultrasound examination was performed. The left lower part of every panel is the coronal view. Red and blue colors indicate blood vessels in the tumor. Responder: (A) Tumor vasculature before the first bevacizumab treatment. The coronal view showed no prominent blood vessels in the mass. (B) The coronal view showed that tumor vessels were enhanced and normalized $20 \mathrm{~h}$ after the delivery of bevacizumab; the 'normalization window' was open. (C) Ten days after bevacizumab with sequential paclitaxel, the coronal view showed tumor shrinkage and that the tumor vessels had returned to inadequate; the window was closed. Non-responder: (D) Tumor vascular patterns prior to the first bevacizumab treatment. (E) No enhancement of tumor vessels $20 \mathrm{~h}$ after bevacizumab delivery.

by an eventual closure of the 'normalization window', at which point the features of normalization are lost (15). The closure of the window may be a result of excessively high or prolonged dosing of anti-VEGF treatment (16). Furthermore, the emergence of anti-VEGF treatment resistance may lead to failed vascular normalization $(16,17)$. Yuan et al reported that at day 1 , but not at day 5 , colon tumor vessels became less permeable and tortuous following the intravenous injection of an anti-VEGF/vascular permeability factor antibody (18). The treatment of the two cases described in the current study, which exhibited a good and rapid response to the BP regimen, were based on this concept.

The present study further provides direct evidence that bevacizumab has a normalization effect in human breast cancer through three-dimensional power Doppler ultrasound in which red and blue colors indicate blood vessels in the tumor. In a typical example of a responder (1 breast cancer patient received neoadjuvant chemotherapy with sequential use of bevacizumab and paclitaxel), the coronal view showed no blood vessels in the mass prior to the first bevacizumab treatment (Fig. 3A); tumor vessels were enhanced and normalized $20 \mathrm{~h}$ after the delivery of bevacizumab, i.e., the window was open (Fig. 3B); 10 days following sequential treatment with bevacizumab and paclitaxel, tumor shrinkage was observed and the vessels returned to being inadequate, i.e., the window was closed (Fig. 3C). In a representative example of a non-responder, identical regimen were used; however, no tumor vessels were enhanced at $20 \mathrm{~h}$ after bevacizumab 
delivery and a poor response was observed (Fig. 3D and E). When retrospectively reviewing previous important clinical trials, including E2100, RIBBON-1, CALGB 40503 (19) and TURANDOT, it was found that bevacizumab added to weekly paclitaxel provides better PFS outcomes (11.3, 10.7, 10.6 and 11 months, respectively) than bevacizumab combined with other regimens, particularly with docetaxel three-weekly (AVADO). The possible explanation is that the elimination half-life of docetaxel is $11 \mathrm{~h}(20)$ and it may miss the 20-24 h normalization window of bevacizumab. Thus, the quantity of docetaxel entering the tumor may be insufficient. However, the half-life of weekly paclitaxel is $6.4 \mathrm{~h}$. This also may miss the opening of the window; however, after 7 days when the second dose is delivered the window may not yet be closed. Thus, the benefit of bevacizumab treatment is obtained. If vascular normalization is the major role of anti-VEGF therapy, a half dose $(5-7.5 \mathrm{mg} / \mathrm{kg})$ of bevacizumab may not only be enough to achieve this purpose as in the current cases, but also decrease the possible side-effects of bevacizumab.

In conclusion, these two cases of MBC have successfully met the hypothetical window of opportunity and demonstrate that using a 20-24 h normalization window may provide the opportunity to enhance the effectiveness of chemotherapy through the aid of bevacizumab. Based on these findings, for the first time to the best of our knowledge, the present study demonstrated the importance of the 20-24 h timing window of vascular normalization in human breast cancer following treatment with bevacizumab. Further clinical trials are necessary to verify this point.

\section{References}

1. Miller K, Wang M, Gralow J, et al: Paclitaxel plus bevacizumab versus paclitaxel alone for metastatic breast cancer. N Engl J Med 357: 2666-2676, 2007.

2. Miles DW, Chan A, Dirix LY, et al: Phase III study of bevacizumab plus docetaxel compared with placebo plus docetaxel for the first-line treatment of human epidermal growth factor receptor 2-negative metastatic breast cancer. J Clin Oncol 28: 3239-3247, 2010.

3. Robert NJ, Diéras V, Glaspy J, et al: RIBBON-1: Randomized, double-blind, placebo-controlled, phase III trial of chemotherapy with or without bevacizumab for first-line treatment of human epidermal growth factor receptor 2-negative, locally recurrent or metastatic breast cancer. J Clin Oncol 29: 1252-1260, 2011.

4. Lang I, Brodowicz T, Ryvo L, et al; Central European Cooperative Oncology Group: Bevacizumab plus paclitaxel versus bevacizumab plus capecitabine as first-line treatment for HER2-negative metastatic breast cancer: Interim efficacy results of the randomised, open-label, non-inferiority, phase 3 TURANDOT trial. Lancet Oncol 14: 125-133, 2013.
5. Brufsky AM, Hurvitz S, Perez E, Swamy R, Valero V, O'Neill V and Rugo HS: RIBBON-2: A randomized, double-blind, placebo-controlled, phase III trial evaluating the efficacy and safety of bevacizumab in combination with chemotherapy for second-line treatment of human epidermal growth factor receptor 2-negative metastatic breast cancer. J Clin Oncol 29: 4286-4293, 2011.

6. Pugh CW and Ratcliffe PJ: Regulation of angiogenesis by hypoxia: Role of the HIF system. Nat Med 9: 677-684, 2003.

7. Jain RK: Normalizing tumor vasculature with anti-angiogenic therapy: A new paradigm for combination therapy. Nat Med 7: 987-989, 2001.

8. Willett CG, Boucher Y, di Tomaso E, et al: Direct evidence that the VEGF-specific antibody bevacizumab has antivascular effects in human rectal cancer. Nat Med 10: 145-147, 2004.

9. Vakoc BJ, Lanning RM, Tyrrell JA, et al: Three-dimensional microscopy of the tumor microenvironment in vivo using optical frequency domain imaging. Nat Med 15: 1219-1223, 2009.

10. Winkler F, Kozin SV, Tong RT, et al: Kinetics of vascular normalization by VEGFR 2 blockade governs brain tumor response to radiation: Role of oxygenation, angiopoietin-1 and matrix metalloproteinases. Cancer Cell 6: 553-563, 2004.

11. Jain RK: Taming vessels to treat cancer. Sci Am 298: 56-63, 2008.

12. Gianni L, Romieu GH, Lichinitser M, et al: AVEREL: A randomized phase III trial evaluating bevacizumab in combination with docetaxel and trastuzumab as first-line therapy for her2-positive locally recurrent/metastatic breast cancer. J Clin Oncol 31: 1719-1725, 2013.

13. Miles DW, de Haas SL, Dirix LY, et al: Biomarker results from the AVADO phase 3 trial of first-line bevacizumab plus docetaxel for HER2-negative metastatic breast cancer. Br J Cancer 108: 1052-1060, 2013.

14. Hegde PS, Jubb AM, Chen D, et al: Predictive impact of circulating vascular endothelial growth factor in four phase III trials evaluating bevacizumab. Clin Cancer Res 19: 929-937, 2013.

15. Goel S, Duda DG, Xu L, et al: Normalization of the vasculature for treatment of cancer and other diseases. Physiol Rev 91: 1071-1121, 2011.

16. Goel S, Wong AH and Jain RK: Vascular normalization as a therapeutic strategy for malignant and nonmalignant disease. Cold Spring Harb Perspect Med 2: a006486, 2012.

17. Giuliano S and Pagès G: Mechanisms of resistance to anti-angiogenesis therapies. Biochimie 95: 1110-1119, 2013.

18. Yuan F, Chen Y, Dellian M, Safabakhsh N, Ferrara N and Jain RK: Time-dependent vascular regression and permeability changes in established human tumor xenografts induced by an anti-vascular endothelial growth factor/vascular permeability factor antibody. Proc Natl Acad Sci USA 93: 14765-14770, 1996.

19. Dickler MN, Barry WT, Cirrincione CT, Ellis MJ, Moynahan ME, Innocenti F, Hurria A, Rugo HS, Lake D, Hahn OM et al: Phase III trial evaluating the addition of bevacizumab to letrozole as first-line endocrine therapy for treatment of hormone-receptor positive advanced breast cancer: CALGB 40503 (Alliance). J Clin Oncol 33 (Suppl): $501,2015$.

20. Bragalone DL (ed): Drug Information Handbook for Oncology. 10th edition. Lexicomp, Hudson, OH, pp501-514, 2012. 